\title{
Determinants of high vitamin A supplementation coverage among pre-school children in Mali: the National Nutrition Weeks experience
}

\author{
Mohamed Ag Ayoya ${ }^{1}$, Mohamed Ag Bendech ${ }^{2, *}$, Shawn K Baker ${ }^{2}$, Fatimata Ouattara ${ }^{3}$, \\ Konaké Alima Diané ${ }^{4}$, Lina Mahy ${ }^{5}$, Lisa Nichols ${ }^{3}$, Aménatou Touré ${ }^{5}$ and Ciro Franco ${ }^{3}$ \\ ${ }^{1}$ Division of Nutritional Sciences, Cornell University, Ithaca, NY, USA: ${ }^{2}$ Helen Keller International, Regional Office for \\ Africa, BP 29.898, Dakar-Yoff, Senegal: ${ }^{3}$ National Technical Assistance/USAID, Abt Associates, Inc., Bamako, \\ Mali: ${ }^{4}$ Direction Nationale de la Santé, Ministère de la Santé, Bamako, Mali: ${ }^{5}$ Helen Keller International, Mali \\ Office, Bamako, Mali
}

Submitted 31 October 2005: Accepted 19 December 2006: First published online 27 February 2007

\begin{abstract}
Objectives: To assess vitamin A supplementation (VAS) coverage of children aged 6-59 months and the factors that favour or limit this coverage during the National Nutrition Weeks in Mali.

Design: Cross-sectional study. Interviews about demographic factors and children's adherence to the vitamin A capsule distribution programme were conducted. Professionals' knowledge of vitamin A and various aspects related to the supplementation strategy were assessed.

Setting: Five regions out of the eight regions in the country, in addition to Bamako District. Three rural communes were selected in three regions to represent rural areas. Subjects: Parents or caregivers of children under 5 years of age, health agents who participated in the weeks, and community and administrative leaders.

Results: At least $80 \%$ of the children received the supplement. More 'traditional' communication channels (town criers, friends and family members) appeared to be more effective in reaching the target groups than modern methods, i.e. radio and television. Mothers' possession of a radio (Pearson $\chi^{2}=5.03 ; P=0.025$ ) and fathers' education (Pearson $\chi^{2}=19.02 ; P<0.001$ ), possession of a radio (Pearson $\chi^{2}=8.93$; $P=0.003$ ) and listening to it (Pearson $\chi^{2}=7.62 ; P=0.006$ ) all appeared to be statistically and significantly associated with children's coverage. In multivariate logistic regression analysis, only the study site (urban/rural) $(P=0.004)$, 'traditional channels' $(P=0.02)$ and fathers' education $(P=0.04)$ were significantly associated with children's coverage. Knowledge about VAS was high among community and administrative leaders, and health professionals. The planning and implementation of activities at the district level were found to be good in general.

Conclusion: National Nutrition Weeks provide a successful example of a periodic VAS strategy with high coverage among children aged 6-59 months in Mali. Campaigns aimed at informing and sensitising populations during the Nutrition Weeks should also target children's fathers.
\end{abstract}

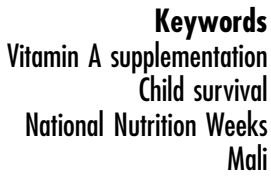

Vitamin A repletion was shown to reduce the mortality of children aged 6-59 months by $23-34 \%$ in areas where vitamin A deficiency (VAD) is prevalent ${ }^{1-4}$. In sub-Saharan Africa, it is estimated that $42 \%$ of children under 5 years of age are at risk of VAD and that adequately controlling this deficiency can avert over 645000 child deaths per year in the region ${ }^{5}$. Twice-yearly vitamin A supplementation (VAS) is a cornerstone of VAD control and child survival in the short term. Strategies for effective delivery of VAS and other low-cost, high-impact child survival services are essential for achieving the Millennium Development Goal of reducing under- 5 mortality by two-thirds by the year 2015, particularly in sub-Saharan Africa.

Various studies have demonstrated the magnitude of VAD in Mali. The 2001 Demographic and Health Survey (DHS) revealed that the prevalence of night blindness in the last pregnancy of mothers of children under 5 is $5.8 \%$ at the national level and $7 \%$ in rural areas where $75 \%$ of Malian women of reproductive age live ${ }^{6}$. This prevalence is higher than the cut-off point of 5\%, indicating that VAD is a public health problem in a population ${ }^{7}$. In order to tackle this situation, Mali developed and applied strategies 
that include a number of combined interventions for control of deficiencies of vitamin A and other micronutrients. The most systematic intervention with national coverage is supplementation of children 6-59 months of age with two doses of vitamin A per year (at 6-month intervals $)^{8}$. Based upon previous successful experiences of linking VAS to National Immunisation Days for poliomyelitis eradication, it was decided to organise twice-yearly weeks during which nutrition activities, particularly VAS, are intensified.

These National Nutrition Weeks (French acronym SIAN) are based on intensifying routine VAS activities during a specific week and/or the organisation of campaigns adapted to local situations. For each SIAN, regional microplanning workshops are organised in each of the eight regions of the country and the capital city. This allows 'custom-made' SIAN in each of the regions that are geographically, ethnically and socially different. It also allows for different and context-specific distribution strategies. For example, in the North where nomadic populations reside, the focus is on mobile teams (camp to camp), whereas in other regions intensified facility-based distribution can be combined with door-to-door campaigns.

We report on results from an evaluation conducted to assess VAS coverage of children aged 6-59 months and the factors that favour or limit this coverage during SIAN. The evaluation was intended to inform local and regional supplementation policies and identify effective prevention and control strategies to strengthen, in future, efforts undertaken by the Mali government and other actors with interests in enhancing the well-being of children.

\section{Subjects and methods}

\section{Design and selection methods}

The sample was a multi-stage cluster sample, with the primary sampling unit being the lowest level administrative unit ('commune' in the regions or 'quartiers' in the District of Bamako). Initially a purposeful selection of regions was done to ensure representation based on geographic distribution, diverse experience in organising SIAN and the presence of internally displaced populations, resulting in selection of the regions of Kayes, Sikasso, Mopti, Tombouctou and Gao, and the District of Bamako. Within each of these zones, a health district was randomly selected (not weighted by population), but excluding the district capital in the case of the five regions. From selected health districts, one 'commune' was randomly selected (not weighted by population) in each region and two 'quartiers' were randomly selected in the District of Bamako (not weighted by population). Within each of these units, 30 households were selected randomly, either from the population list of the 2001 census (in the case of Bamako) or by a random start in the centre of the 'commune' and moving from house to house in a rightward direction until 30 households were surveyed (in the case of the regions). Within households, one child aged 6-59 months was randomly selected and the parents and/or caregiver interviewed. In total, 210 households were selected. Only health workers (medical doctors, nurses, nurse assistants, technicians, etc.) who participated in the weeks and were present on the day of interviews were selected. Local leaders in the selected units (mayor, mayor's counsellors, chiefs of the village, members of health community associations and leaders of women's groups) and administrative authorities (chiefs) who had these units under their jurisdiction were also systematically interviewed.

\section{Study subjects}

The study population consisted of mothers and fathers or caregivers of children under 5 years of age in each selected household. Health agents that were involved in SIAN, community leaders, and local health and administrative authorities of the survey areas were also included.

\section{Methods}

Structured questionnaires were used to collect the data, and interviews were conducted by trained and experienced investigators. Coverage was assessed by asking mothers and fathers or caregivers whether their child had received a vitamin A capsule during SIAN. Sample capsules of 100000 IU and 200000 IU were concurrently shown to the person who brought the child to the health centre or witnessed his/her coverage at home to facilitate recall and avoid confusion with other products. Health and community agents and administrative leaders were also interviewed on several aspects of VAS. More specifically, health agents were interviewed on their knowledge on VAS (target groups, adequate dosages for each group, per annum frequency of administering vitamin A capsules and health benefits), the distribution strategies used and their level of involvement in the organisation of the Nutrition Weeks. Community and administrative leaders were asked about VAS target groups, their level of involvement in the organisation of the SIAN and the role they played in mobilising populations for the event. All respondents were invited to give their judgement about the organisation of SIAN and to suggest ways for improving them in the future. Experienced supervisors followed the teams regularly and reviewed questionnaires at the end of each day to ensure that the collected data were of high quality.

\section{Statistical analyses}

All the study's data were entered and analysed using Excel 2000 and SPSS for Windows version 11.5 (SPSS Inc.). Frequencies of general sociodemographic characteristics of the parents were computed and the proportion of children said to receive VAS was estimated. This proportion was broken down according to the parents' 
information sources and compared across fathers and mothers given their education, possession of a radio and whether they listen to that source of information. The effectiveness of the various communication channels used to inform populations was also evaluated. Children's coverage was estimated further using their parents' decision-making role within the household. Other factors which are likely to influence coverage, such as leaders' and health professionals' knowledge about vitamin A, their role and involvement in SIAN, vitamin A capsule availability and delivery mechanisms, preparatory training sessions for health professionals, and the planning and implementation of activities within the districts, were also assessed.

Comparisons among categorical variables were tested by $\chi^{2}$ statistics. Significant relationships were assessed by multivariate logistic regression models that included fathers' and mothers' education, possession of a radio and listening to it, their access to the information through other communication channels and the study sites (urban vs. rural). These factors (independent variables) were examined against coverage (dependent variable). They were chosen because of their high likelihood to influence coverage, and hence were expected to be its best predictors. A $P$-value $<0.05$ was considered significant.

\section{Results}

Table 1 summarises selected sociodemographic characteristics of children's parents who were interviewed.

The proportion of children who received vitamin A capsules during the SIAN according to their parents is shown in Table 2. At least $80 \%$ of the children whose parents were interviewed were reported to have received VAS.

Table 2 also gives the coverage of children based upon the household decision-maker with regards to seeking VAS for the child. Fathers clearly played an important role in children's access to the supplement during these events.

Children's coverage differed according to mothers' and fathers' education status and their possession of and listening to a radio. Coverage also differed according to parents' information source concerning SIAN (Fig. 1) and the study sites (92\% in urban vs. 69\% in rural areas). More

Table 1 Selected sociodemographic characteristics of children's parents

\begin{tabular}{lcc}
\hline Descriptive characteristics & $\begin{array}{c}\text { Mothers' } \\
\text { frequency } \\
(n=210)\end{array}$ & $\begin{array}{r}\text { Fathers' } \\
\text { frequency } \\
(n=210)\end{array}$ \\
\hline Received an education & $43 \%$ & $57 \%$ \\
Attended high school & $10 \%$ & $30 \%$ \\
$\begin{array}{l}\text { Attended university } \\
\text { Occupation }\end{array}$ & $3 \%$ & $7 \%$ \\
$\quad$ Merchants & $11 \%$ & $12 \%$ \\
$\quad$ Civil servants & $7 \%$ & $29 \%$ \\
\hline
\end{tabular}

Table 2 Vitamin A supplementation (VAS) coverage and principal decision-maker in seeking VAS for the child as reported by mothers and fathers

\begin{tabular}{ll}
\hline Parents' report & $\begin{array}{c}\text { Frequency } \\
(n=210)\end{array}$ \\
\hline Received vitamin $\mathrm{A}$ capsule & \\
$\quad$ Mothers $(95 \% \mathrm{Cl})$ & $83 \%(67-95)$ \\
Fathers $(95 \% \mathrm{CI})$ & $80 \%(61-93)$ \\
Made the decision to seek VAS for the child & \\
$\quad$ Mothers & $23 \%$ \\
Fathers & $77 \%$ \\
\hline
\end{tabular}

$\mathrm{Cl}$ - confidence interval.

'traditional' communication channels (town criers, friends and family members) appeared to be more effective in reaching the target groups than modern methods, i.e. radio and television. This was expressed more frequently by women living in rural than in urban areas (Pearson $\chi^{2}=6.52 ; P=0.011$ ). The advantage of these 'traditional' channels was also stated by the majority of community and administrative leaders (81\%; i.e. 34/42) and health professionals (63\%, i.e. 35/56). There were no statistically significant differences between the coverage of children born to educated mothers and those born to non-educated mothers (Pearson $\chi^{2}=1.61 ; P=0.205$ ). Similarly, no statistically significant differences were found between the coverage of children whose mothers listen to the radio and that of those whose mothers do not (Pearson $\chi^{2}=2.86$; $P=0.091)$. On the contrary, mothers' possession of a radio (Pearson $\chi^{2}=5.03 ; P=0.025$ ) and fathers' education (Pearson $\chi^{2}=19.02 ; P<0.001$ ), possession of a radio (Pearson $\chi^{2}=8.93 ; P=0.003$ ) and listening to it (Pearson $\chi^{2}=7.62 ; P=0.006$ ) all appeared to be statistically and significantly associated with children's coverage. In multivariate logistic regression analysis, only the study site (urban vs. rural) $(P=0.004)$, other communication channels (i.e. traditional channels) through which the information was received $(P=0.02)$ and fathers' education $(P=0.044)$ were significantly associated with children's coverage (Table 3 ).

Knowledge about VAS target groups (children 6-59 months of age and postpartum women) was high among

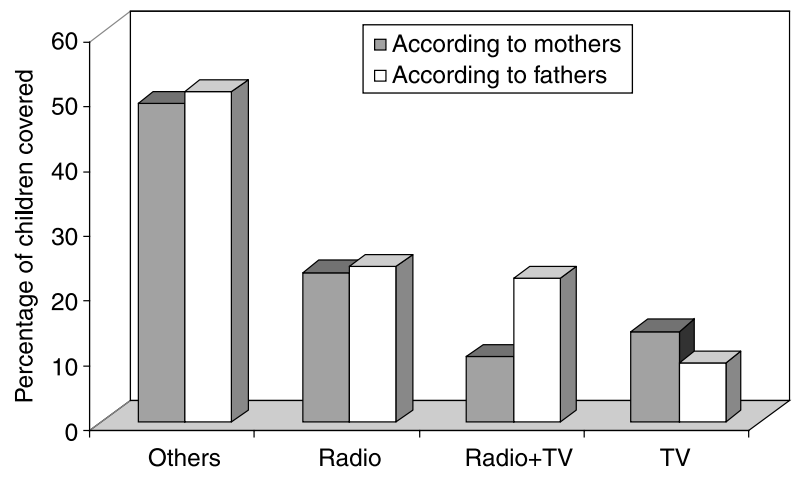

Fig. 1 Vitamin A supplementation coverage by parents' principal information sources. Others include town criers, family members and friends, i.e. 'traditional channels' 
Table 3 Results of the regression analysis with children's coverage as the dependent variable

\begin{tabular}{|c|c|c|c|c|c|c|}
\hline Predictors & B & SE & Wald & d.f. & Significance & $\operatorname{Exp}(B)$ \\
\hline Father's education & 0.394 & 0.472 & 0.698 & 1 & 0.044 & 1.483 \\
\hline Mother's education & -0.564 & 0.483 & 1.367 & 1 & 0.242 & 0.569 \\
\hline Traditional channel & 0.160 & 0.068 & 5.568 & 1 & 0.018 & 1.173 \\
\hline Possession of a radio & 0.390 & 0.711 & 0.300 & 1 & 0.584 & 1.476 \\
\hline Listening to the radio & -0.041 & 0.662 & 0.004 & 1 & 0.951 & 0.960 \\
\hline Study site (urban/rural) & 1.479 & 0.510 & 8.425 & 1 & 0.004 & 4.390 \\
\hline Constant & -6.403 & 1.081 & 35.114 & 1 & 0.000 & 0.002 \\
\hline
\end{tabular}

SE - standard error; d.f. - degrees of freedom.

community and administrative leaders (79\%) and health professionals (88\%). Similarly, high proportions of community and administrative leaders (57\%) and health professionals (78\%) knew the frequency with which vitamin A capsules should be given to children under 5 years old (i.e. twice a year at 6-month intervals). Furthermore, 98 and $88 \%$ of health professionals knew, respectively, that there exists an age-dose relationship in supplementing children with vitamin A and that this supplement is good for the sight and protects children against illnesses.

Both the leaders and the health professionals were involved in sensitising and mobilising communities for the events. They also were jointly involved in planning and implementing the activities within their respective districts, even though community institution leaders felt that their involvement was only partial. Health professionals and extension agents were those responsible for administering the supplement to the children.

The availability of vitamin A capsules within health services at the time of the event was constant according to $86 \%$ of the health professionals. Stock-outs were reported in three districts, and it was found that vitamin A capsule availability outside SIAN for routine administration to children and postpartum women is very limited and not always ensured. Capsules were delivered through various mechanisms including distribution within existing health centres (fixed strategy) and advanced/mobile strategies allowing the health agents to reach beneficiaries in their houses or at schools in villages that do not have health facilities. Many more children were covered through the advanced/mobile distribution strategy than through the fixed strategy.

Only 59\% of health agents involved in SIAN received a preparatory training session. The majority of agents who were trained (91\%) found the training appropriate and valuable. Those who said the opposite found it to be too short and/or speedy.

Tally sheets were made available to the districts on time. Very few health agents (7\%) reported having difficulties in completing them. Among these were three supervisors and one distributor.

The planning and implementation of activities at the district level were found to be good in general by the majority of respondents. However, they suffered from a number of weaknesses, among which are: lack of enough personnel to ensure wider distribution of capsules; insufficient working tools (scissors for example); insufficient logistic resources to guarantee the mobilisation of populations and the delivery of capsules to suburban and remote areas; diffusion of less explicit messages through radio and television; partial involvement of community and administrative leaders; lack of training of many health agents involved in SIAN; vitamin A capsule stock-outs in some districts; insufficient and late availability of funds, and the re-scheduling of SIAN.

\section{Discussion}

In this study, VAS coverage of children aged 6-59 months during the SIAN and the factors that favour or limit a high VAS coverage during those weeks were assessed. The results indicate successful endeavours and highlight key lessons learned, which need to be capitalised on.

The proportion of children who received the supplement according to their mothers was high, i.e. 83\%. This coverage is lower than but close to the $96 \%$ obtained through tally sheets? This high coverage is very probably the result of an overall satisfactory organisation, planning and implementation of the Nutrition Weeks' activities at national and local levels, coupled with the committed support of development partners and a good mobilisation of recipients. These factors were reported by Aguayo et $a .^{10}$ in Niger, a neighbouring country, by Houston ${ }^{11}$ in Ghana, Zambia and Nepal, and by Mora and Bonilla ${ }^{12}$ in Nicaragua as key to the success of similar events. This coverage may have been underestimated since it would drop if capsules were not available, which was the case in some districts, or if caregivers were not bringing children to be dosed, which was also encountered in others. Also, accurate determination of age is extremely difficult in many African societies. Therefore, children older than 59 months may have been considered as part of the target group and, as a consequence, may have received the supplement. This latter fact also could have contributed to the occurrence of vitamin A capsule stock-outs. However, this is a difficult situation to deal with. In fact, refusing to dose a child (among two or three for example) brought by a parent to the site of distribution may be frustrating for both the parent and the agent. It may discourage the 
parent from bringing children during a subsequent event or force the agent to administer the supplement and thus count the child among the target group, which will result in overestimating the numerator. Therefore, there is a need to revise currently used tally sheets and add a new column for children older than 59 months in order to compute confidently an accurate coverage. This is even more important because dosing children in this older age group may not need more resources, and may actually be beneficial to their health status in this region of the world.

The study showed that the use of radio and television as communication channels to inform, sensitise and mobilise populations is good, but not as effective as the traditional sources such as town criers and other social networks, especially in rural areas. This is in agreement with results obtained by Houston ${ }^{11}$ in Ghana. These findings suggest that more attention and resources should focus on the use of information sources using traditional channels and networks, and should make more effective use of the radio networks. Television is not accessible to everyone and, even when it is, it may not be watched regularly. In Mali, there is a network of 144 public, private and community radio stations that ensure large and widespread information dissemination at the national level. The fact that only two of these were used in each area to disseminate the information about the Nutrition Weeks, instead of all of them, probably explains why they were not considered as an essential information source. Therefore, it may be more strategic to maximise their use in the future. Town criers, on the other hand, can go inside public gathering areas (markets for example) and disseminate the information easily and in a comprehensive language and a culturally acceptable manner. Furthermore, swift information exchange among family members and social networks is very common in Mali.

Challenges limiting the success of the SIAN were identified in this study. They all need to be addressed effectively in order to ensure better distribution of vitamin $\mathrm{A}$ and better coverage. More specifically, all those in charge of administering the supplement must receive refresher training prior to the event. This is important because a trained worker is a sure, motivated and confident resource in accomplishing required tasks. National health services and non-governmental organisations working in nutrition in Mali have significant experience in training supplementation and supervision agents at all levels using the cascade approach. This is a strategy during which master trainers are trained at the central level, and these train other trainers per health district who then train the district supplementation and supervision agents. This acquired experience is a valuable asset that should be used to fill the current training gap. Availability of vitamin A capsules and a timely provision of adequate and sufficient resources to ensure their appropriate use and delivery should be a priority. This is vital because stock-outs discourage and demobilise parents, and inadequate work tools discourage providers. In addition, vitamin A capsules should be available permanently within health centres to make sure that needy postpartum women and children receive them, as appropriate. This is a necessary first step to ensure protection of children aged 6-59 months against VAD and severe childhood illnesses ${ }^{13}$. However, it is important to stress that this step is not sufficient because of known limitations of VAS as a strategy for the control of VAD. For example, it was suggested that the protective effect and the biological impact of vitamin A capsules does not last for a full 6-month period ${ }^{14}$. Furthermore, VAS is considered to be a short-term strategy that is completely dependent on the availability of capsules. Therefore, efforts to increase dietary vitamin A intakes in this group should be actively and continuously pursued, because this represents a sustainable solution to combating the deficiency in a population ${ }^{14,15}$. Traditional and elected leaders and administrators should be closely involved. This is crucial because they have a unique potential to mobilise populations. Furthermore, such involvement will ensure more transparency and encourage these leaders to be more engaged in the health of their people in the growing context of decentralisation.

The most interesting finding is that fathers' education, instead of that of the mothers, appeared to be strongly and significantly associated with children's coverage (Pearson $\chi^{2}=19.02 ; \quad P<0.001$, and $P=0.044$ in multivariate analysis). This suggests that fathers, especially those with a lower level of education, should be considered as a priority target for children's nutrition/health education and communication of messages. The finding was unexpected but not entirely surprising because, even though fathers in Mali usually do not directly provide care for children, mothers always consult them before they make any decisions regarding children's health and nutrition. In that respect, it would make sense that educated fathers, who may be more aware of the health benefits of vitamin A, would encourage the receipt of VAS by their children. This cultural 'male power' may dilute an existing but not found effect of mothers' education on the coverage of children in this study. Moreover, the chances of an educated woman being married to a non-educated man in this context, and consequently leading to the decision to let the child receive supplements, are also very low. In fact, the opposite would be common in Mali. Finally, an important limitation for not finding a statistically significant effect of mothers' education could be the small sample size of educated mothers in this study, which increases the risk of type II error.

The study has a number of limitations that should be considered in interpreting the results. The selection of households starting from the centre of the commune may introduce some bias, as more remote households may not be included, especially if the commune is large and spread out. In addition, the lack of sample weighting for the area 
population also might have constrained the sampling frame.

\section{Conclusions and recommendations}

The National Nutrition Weeks provide a successful example of a periodic VAS strategy with high coverage among children aged 6-59 months in Mali. Fathers' education and the use of town criers as a means of informing and sensitising populations had a positive and significant effect on this success. These weeks should be complemented by a steady availability of vitamin A capsules within health services for their routine distribution, and efforts to increase dietary vitamin A intakes should be pursued. They should also serve as an excellent occasion to strengthen relationships among communities, health professionals, and community and administrative leaders. This will offer a good opportunity to improve and sustain the coverage attained among children and contribute to reaching other target groups at the same time.

Our results allow the following recommendations, which could be extended to other settings that are similar to Mali:

- Increasing the number of trained health agents.

- Capitalising much more on the use of community radio stations and maximising the use of traditional communication channels.

- Adjusting the communication strategy by improving fathers' involvement as they play an important role in the decision-making process.

- Improving traditional leaders' involvement in the organisation of SIAN.

- Improving the availability of vitamin A capsules during SIAN and for routine services.

- Modifying the tally sheets by adding in a new column for children older than 59 months who may receive the supplements during SIAN in order to avoid their inclusion in the numerator and hence bias the reported coverage.

\section{Acknowledgements}

Sources of funding: This study was funded by USAID agreement No. 688-C-00-04-00011-00 USAID/Abt Associates Inc./HKI/JHU-CCP/IBEX/Intrah/SDSS and the Micronutrient Initiative with funding from the Canadian International Development Agency.

Conflict of interest declaration: The authors declare no conflict of interest.

M.A.A., M.A.B., S.K.B. and F.O. designed the study. K.A.D. and A.T. contributed to the selection of the regions. M.A.A. oversaw the data collection, did the statistical analyses and prepared the first draft of the manuscript with inputs from M.A.B., S.K.B., F.O. and L.N. All authors reviewed the first draft and approved the final version.
Acknowledgements: Our sincere appreciation goes to the mothers, fathers and caregivers, the health agents, and the community and administrative leaders of the selected regions for participating in this study. The authors thank Professor Cutberto Garza, Boston College, USA for his critical review of an earlier draft.

\section{References}

1 Beaton GH, Martorell R, Aronson KJ, Edmonston B, McCabe $\mathrm{G}$, et al. Effectiveness of Vitamin A Supplementation in the Control of Young Child Morbidity and Mortality in Developing Countries. ACC/SCN State-of-the-Art Series: Nutrition Policy Discussion Paper No. 13. Geneva: United Nations, 1993.

2 Fawzi WW, Chalmers TC, Herrera MG, Mosteller F. Vitamin A supplementation and child mortality: a meta-analysis. Journal of the American Medical Association 1993; 269: 898-903.

3 Glaziou PP, Mackerras DEM. Vitamin A supplementation and infectious disease: a meta-analysis. British Medical Journal 1993; 306: 366-70.

4 Tonascia JA. Meta-analysis of published community trials: impact of vitamin A on mortality. Proceedings of the Bellagio Meeting on Vitamin A Deficiency and Childhood Mortality. New York: Helen Keller International, 1993.

5 Aguayo V, Baker SK. Vitamin A deficiency and child survival in sub-Saharan Africa: a reappraisal of challenges and opportunities. Food and Nutrition Bulletin 2005; 26: 348-55.

6 Ministère de la Santé (CPS/MS), Direction Nationale des Statistiques et de l'Informatique (DNSI) and ORC Macro. Enquête Démographique et de Santé (EDSM-III) Mali 2001. Cellule de Planification et de Statistique. Calverton, MD: CPS/MS, DNSI and ORC Macro, 2002.

7 Christian P. Recommendations for indicators: night blindness during pregnancy - a simple tool to assess vitamin A deficiency in a population. Journal of Nutrition 2002; 132: 2884S-8S

8 Division Nutrition, Direction Nationale de la Santé, Ministère de la Santé du Mali. Stratégie Nationale de Supplémentation en Vitamine A et fer/folate. Bamako: Ministère de la Santé, 2003.

9 Division Nutrition, Direction Nationale de la Santé, Ministère de la Santé du Mali. Résultats des SIAN, éditions 1 et 2. Bamako: Ministère de la Santé, 2004.

10 Aguayo VM, Baker SK, Crespin X, Hamani H, Mamadoul Taibou A. Maintaining high vitamin A supplementation coverage in children: lessons from Niger. Food and Nutrition Bulletin 2005; 26: 26-31.

11 Houston R. Why They Work: An Analysis of Three Successful Public Health Interventions. Vitamin A Supplementation Programs in Ghana, Nepal and Zambia. Arlington, VA: MOST Project, 2003.

12 Moré JO, Bonilla J. Successful vitamin A supplementation in Nicaragua. Sight and Life Newsletter 2002; 3: 75-9.

13 Bryce J, Black RE, Walker N, Bhutta ZA, Lawn JE, Steketee RW. Can the world afford to save the lives of 6 million children each year? Lancet 2005; 365: 2193-200.

14 Bloem MW, Hye A, Wijnroks M, Ralte A, West KP Jr, Sommer A. The role of universal distribution of vitamin A capsules in combating vitamin A deficiency in Bangladesh. American Journal of Epidemiology 1995; 142: 843-55.

15 Delisle H, Zagré N, Bakari S, Codjia P, Zendong R. Des solutions alimentaires à la carence en vitamine A. Food, Nutrition and Agriculture 2003; 32: 40-9. 